\title{
Research of General Fuzzy Integral Sliding Mode Controller Based on Complex Networks
}

\author{
Dongsheng Yang ${ }^{1}$, Bingqing $\mathrm{Li}^{1,2}, \mathrm{He} \mathrm{Jiang}^{1}$, Xingyu Liu ${ }^{2}$ and Yihe Wang ${ }^{3}$ \\ 1 College of Information Science and Engineering Northeastern University, \\ Liaoning Province, China \\ 2 State Grid Liaoning Electric Power Supply Co., Ltd., Liaoning Province, China \\ 3 State Grid Shenyang Electric Power Supply Co., Ltd., Liaoning Province, China
}

\begin{abstract}
A fuzzy integral sliding mode control method based on the finite time stable theory and integral sliding mode control theory is proposed to solve a class of complex network problems. The complex networks are expressed by T-S fuzzy models with bounded approximation errors by using the approximation capability of T-S fuzzy models. The paper proposes fuzzy dynamic integral sliding mode control scheme and the scheme is designed for the complex networks based on their T-S fuzzy approximation models. The simulation result shows that the trajectories of the closed-loop control system are kept on the sliding surface almost since the initial time. And the sliding motion of the closed-loop control system involving the original nonlinear system can be shown to be semi globally asymptotically stable. The controller can make system convergence in finite time and reduce the system buffeting effectively.
\end{abstract}

Keywords: Fuzzy, complex networks, integral sliding mode, nonlinear system

\section{Introduction}

Sliding mode control is also called variable structure control. It is under the action of the sliding mode controller, and controlled system comes into high frequency switched state to force it move continuously on the sliding surface. Eventually it will converge to the desired sliding mode along the sliding mode surface so as to achieve the goal of control. Sliding mode control is a very effective method in dealing with uncertain high-order nonlinear dynamic system [1]. It gives expression to the uncertain nonlinear system's smaller error, external disturbance's strong robustness and the simple algorithm design. But it can produce high frequency chattering of sliding mode in the actual sliding mode control system due to the influence of the non-ideal effects. With the development of complex networks in recent years, complex network appears in various kinds of actual system. A complex network composes of many nodes and every node connects with each other. It is high dimension, strong coupling and uncertainty, so the traditional single node control theory is difficult to solve multiple nodes complex network system's analysis and design problems. In the past ten years, many researches are based on the analysis of the T - S fuzzy model in order to study the issue of complex nonlinear systems and complex networks. A series of fuzzy integral sliding mode control scheme of the nonlinear system can be put forward with this model, because it provides the advantage of traditional control theory and technology.

This paper puts forward a new kind of fuzzy dynamic integral sliding mode control method to realize the stability of the nonlinear system. The key is that the integral sliding surface depends on both of the system state vector $\mathrm{x}$ and control input vector $\mathrm{u}$. The sliding motion can be guaranteed by solving LMI matrix, and 
the integral sliding surface along with the dynamic sliding-mode controller [2] can be obtained.

\section{Problem Descriptions and the Preliminary Knowledge}

The ith child node of the complex network can be expressed in formula (1):

$$
d x_{i}(t)=f\left(x_{i}(t), u_{i}(t)\right)
$$

Consider a class of complex network composed of $\mathrm{N}$ child nodes $S_{i}, i=1,2 \cdots, \mathrm{N}$. Each rule of child node $S_{i}$ can use T - S fuzzy model to describe:

$$
\begin{aligned}
& \text { If } \zeta_{i 1} \text { is } M_{i 1}{ }^{l} \text { and } \ldots \text { and } \zeta_{i g_{i}} \text { is } M_{i g_{i}}{ }^{l} \text {; Then } \\
& \qquad \begin{aligned}
d x_{i}(t) & =F_{i}^{l} x_{i}(t)+G_{1 i}{ }^{l} w_{i}(t)+G_{2 i}{ }^{l} u_{i}(t)+\Omega_{i}(t) \\
\Omega_{i}(t) & =\sum_{j=1 j \neq i}^{N} H_{j i} x_{j}(t) \\
z_{i}(t) & =K_{i}^{l} x_{i}(t)+L_{i} u_{i}(t) \\
y_{i}(t) & =K_{y i}{ }^{l} x_{i}(t)+L_{y i}{ }^{l} w_{i}(t), i=1,2 \cdots N, l=1,2 \cdots r_{i}
\end{aligned}
\end{aligned}
$$

$F_{i}^{l}, G_{1 i}{ }^{l}, G_{2 i}{ }^{l}, K_{i}{ }^{l}, L_{i}$ represent the rule node matrix of the ith child node. $H_{j i}$ represents the interconnection matrix of the ith child node and the jth child node. $x_{i} \in R^{n i}, u_{i} \in R^{m i}, w_{i}, z_{i}, y_{i}$ respectively represent the system state, control, disturbance, measurable output and the output vector. If adopting the single point blurred, the product of reasoning and center average solution of the blur, the above formula can be represented as:

$$
\begin{aligned}
& d x_{i}(t)=\sum_{l=1}^{\eta_{i}} \eta_{i}^{l}\left(\zeta_{i q}(t)\right)\left(F_{i}^{l} x_{i}(t)+G_{1 i}{ }^{l} w_{i}(t)+G_{2 i}{ }^{l} u_{i}(t)+\sum_{j=1 \neq j}^{N} H_{j i} x_{j}(t)\right) \\
& z_{i}(t)=\sum_{l=1}^{r_{i}} \eta_{i}^{l}\left(\zeta_{i q}(t)\right)\left(K_{i}^{l} x_{i}(t)+L_{i} u_{i}(t)\right) \\
& y_{i}(t)=\sum_{l=1}^{r_{i}} \eta_{i}^{l}\left(\zeta_{i q}(t)\right)\left(K_{y i}{ }^{l} x_{i}(t)+L_{y i}{ }^{l} w_{i}(t)\right) \\
& \gamma_{i}^{l}\left(\zeta_{i q}(t)\right)=\prod_{q=1}^{g_{i}} M_{i q}{ }^{l}\left(\zeta_{i q}(t)\right), \quad \eta_{i}^{l}\left(\zeta_{i q}(t)\right)=\frac{\gamma_{i}^{l}\left(\zeta_{i q}(t)\right)}{\sum_{l=1}^{r_{i}} \gamma_{i}{ }^{l}\left(\zeta_{i q}(t)\right)}
\end{aligned}
$$

$\zeta_{i 1}, \cdots, \zeta_{i i_{i}}$ is antecedent variable, $M_{i q}{ }^{l}\left(\zeta_{i q}(t)\right)$ is membership of $M_{i q}{ }^{l}$. It is observed that $\gamma_{i}^{l}\left(\zeta_{i q}(t)\right) \geq 0, \eta_{i}^{l}\left(\zeta_{i q}(t)\right) \geq 0, \sum_{l=1}^{r_{i}} \eta_{i}^{l}\left(\zeta_{i q}(t)\right)=1, i=1, \cdots, J, l=1, \cdots r_{i}, r_{i}$ is ith child node rule's amount. For any complex network, $f\left(x_{i}(t), u_{i}(t)\right)$ is in the interval of $X \times U$ and $f(0,0)=0$. There exist s positive constant $\varepsilon_{f}$. So the T-S fuzzy model is described as follows:

$$
\hat{f}\left(x_{i}, u_{i}\right)=\sum_{l=1}^{r_{i}} \eta_{i}{ }^{l}\left(\zeta_{i q}(t)\right)\left(F_{i}^{l} x_{i}(t)+G_{1 i}{ }^{l} w_{i}(t)+G_{2 i}{ }^{l} u_{i}(t)+\sum_{j=1 j \neq i}^{N} H_{j i} x_{j}(t)\right)
$$




$$
\begin{gathered}
f\left(x_{i}, u_{i}\right)=\hat{f}\left(x_{i}, u_{i}\right)+\Delta E\left(x_{i}, u_{i}\right) \\
=\sum_{l=1}^{r_{i}} \eta_{i}^{l}\left(\zeta_{i q}(t)\right)\left(\left(F_{i}^{l}+\Delta F_{i}^{l}\right) x_{i}(t)+G_{1 i}{ }^{l} w_{i}(t)+\left(G_{2 i}{ }^{l}+\Delta G_{2 i}{ }^{l}\right) u_{i}(t)+\sum_{j=1 j \neq i}^{N} H_{j i} x_{j}(t)\right) \\
\|\Delta E(x, u)\|=\left\|\sum_{l=1}^{r_{i}} \eta_{i}{ }^{l}\left(\zeta_{i q}(t)\right)\left[\Delta F_{i}^{l}, \Delta G_{2 i}{ }^{l}\right] *\left[X^{T}, U^{T}\right]^{T}\right\| \\
\left\|\Delta F_{i}^{l}, \Delta G_{2 i}{ }^{l}\right\| \leq \varepsilon_{f}
\end{gathered}
$$

According to (6),(9), (11), the approximation error upper bound value can obtain small enough by selecting enough large values of fuzzy rules in the T-S fuzzy model[3]. Through the analysis of the approximation error, one can get:

$$
\dot{x}_{i}(t)=\sum_{l=1}^{r_{i}} \eta_{i}{ }^{l}\left(\zeta_{i q}(t)\right)\left(\left(F_{i}^{l}+\Delta F_{i}^{l}\right) x_{i}(t)+G_{1 i}{ }^{l} w_{i}(t)+\left(G_{2 i}{ }^{l}+\Delta G_{2 i}{ }^{l}\right) u_{i}(t)+\sum_{j=1 j \neq i}^{N} H_{j i} x_{j}(t)\right)
$$

\section{The Design of Dynamic Integral Sliding Mode Control}

\subsection{Design of the Dynamic Sliding Mode Controller and the Integral Sliding Surface}

For the provided nonlinear model(1), (6), the following fuzzy integral sliding surface is designed as follows:

$$
\left\{\begin{array}{l}
S_{i}(t)=A_{i}^{x} d_{i}(t)+A_{i}^{u} e_{i}(t)-\int_{0}^{t} \sum_{l=1}^{r_{i}} \eta_{i}^{l}\left(\zeta_{i q}(t)\right) A_{i}^{x}\left(F_{i}^{l} x_{i}(\phi)+G_{2 i}^{l} u_{i}(\phi)\right) d \phi-\int_{0}^{t} \sum_{l=1}^{r_{i}} \eta_{i}^{l}\left(\zeta_{i q}(t)\right) A_{i}^{u}\left(E_{i}^{l} x_{i}(\phi)+H_{i}^{l} u_{i}(\phi)\right) d \phi \\
d_{i}(t)=x_{i}(t)-x_{i}(0) \\
e_{i}(t)=u_{i}(t)-u_{i}(0)
\end{array}\right.
$$

$A_{i}^{x} 、 A_{i}^{u} 、 E_{i}^{l} 、 H_{i}^{l}$ respectively describe $\mathrm{m} * \mathrm{n} 、 \mathrm{~m} * \mathrm{~m} 、 \mathrm{~m} * \mathrm{n} 、 \mathrm{~m} * \mathrm{~m}$ matrix.

Theorem: Consider the nonlinear system in formula (1) and the fuzzy system in formula (6). One can use the following fuzzy controller:

$$
\begin{aligned}
& \text { If } \zeta_{i 1} \text { is } M_{i 1}^{l} \text { and ...and }{ }^{\zeta_{i g_{i}}} \text { is } M_{i g_{i}}{ }^{l} \text {; Then } \\
& \dot{u}_{i}(t)=E_{i}^{l} x_{i}(t)+H_{i}^{l} u_{i}(t)-G_{1 i}^{l} w_{i}(t) A_{i}^{u^{1-1}} A_{i}^{x}-\sum_{j=1 j \neq i}^{N} H_{j i} x_{j}(t) A_{i}^{u^{1}} A_{i}^{x}-\left(\omega+v_{i}(t)\right) A_{i}^{u^{1}} \operatorname{sgn}\left(s_{i}(t)\right), i=1, \cdots, J, l=1, \cdots r_{i}
\end{aligned}
$$

the fuzzy controller using fuzzy rules can also be described as follows :

$$
\begin{gathered}
\dot{u}_{i}(t)=\sum_{l=1}^{r_{i}} \eta_{i}^{l}\left(\zeta_{i q}(t)\right)\left[E_{i}^{l} x_{i}(t)+H_{i}^{l} u_{i}(t)-G_{l i}^{l} w_{i}(t) A_{i}^{u^{1}} A_{i}^{x}-\sum_{j=1 j \neq i}^{N} H_{j i} x_{j}(t) A_{i}^{u^{-1}} A_{i}^{x}\right]-\left(\omega+v_{i}(t)\right) A_{i}^{u^{-1}} \operatorname{sgn}\left(s_{i}(t)\right) \\
v_{i}(t)=\varepsilon_{f}\left\|A_{i}^{x}\right\|\left\|\left[x_{i}^{T}(t), u_{i}^{T}(t)\right]^{T}\right\|
\end{gathered}
$$

$\omega$ is a positive constant, the closed-loop control system trajectory can keep on the sliding surface almost since the finite time by using the above integral controller, Furthermore constructing Lyapunov function[4] for the subsystem function to prove:

$$
V(t)=\frac{1}{2} \sum_{i=1}^{N} s_{i}(t) s_{i}^{T}(t)
$$




$$
\dot{s}_{i}(t)=A_{i}^{x} \dot{x}_{i}(t)+A_{i}^{u} \dot{u}_{i}(t)-\sum_{l=1}^{r_{i}} \eta_{i}^{l}\left(\zeta_{i q}(t)\right) A_{i}^{x}\left(F_{i}^{l} x_{i}(t)+G_{2 i}^{l} u_{i}(t)\right) d t-\sum_{l=1}^{r_{i}} \eta_{i}^{l}\left(\zeta_{i q}(t)\right) A_{i}^{u}\left(E_{i}^{l} x_{i}(t)+H_{i}^{l} u_{i}(t)\right) d t
$$

Then from(14), (17), (20)one has :

$$
\begin{aligned}
& \dot{s}_{i}(t)=\sum_{l=1}^{r_{i}} \eta_{i}^{l}\left(\zeta_{i q}(t)\right) A_{i}^{x}\left(\Delta F_{i}^{l} x_{i}(t)+\Delta G_{2 i}{ }^{l} u_{i}(t)\right) d t-\left(\omega+v_{i}(t)\right) \operatorname{sgn}\left(s_{i}(t)\right) \\
& \dot{V}(t)=\sum_{i=1}^{N} \dot{s}_{i}(t) s_{i}^{T}(t)=\sum_{i=1}^{N}\left\{s_{i}^{T}(t)\left[\sum_{l=1}^{r_{i}} \eta_{i}^{l}\left(\zeta_{i q}(t)\right) A_{i}{ }^{x}\left(\Delta F_{i}^{l} x_{i}(t)+\Delta G_{2 i}{ }^{l} u_{i}(t)\right) d t-\left(\omega+v_{i}(t)\right) \operatorname{sgn}\left(s_{i}(t)\right)\right]\right\} \\
& \quad \leq \sum_{i=1}^{N}\left(\left\|s_{i}(t)\right\| \varepsilon_{f}\left\|A_{i}{ }^{x}\right\|\left\|\bar{x}_{i}(t)\right\|-\left(\omega+v_{i}(t)\right)\left\|s_{i}(t)\right\|\right)
\end{aligned}
$$

Then from (18), (19), (22)one has :

$$
\dot{V}(t) \leq-\sum_{i=1}^{N} \omega\left\|s_{i}(t)\right\|=-\sum_{i=1}^{N} \omega \sqrt{2 V(t)}
$$

Through the above formula one can prove that the system's trajectory[5] can keep on the sliding surface in the limited time, and the above argument has given the relevant proof. So define other sliding surface equation in order to describe the trajectory conveniently.

$$
\begin{gathered}
s_{i}(t)=\bar{A}\left\{\bar{f}_{i}(t)-\int_{0}^{t} \sum_{l=1}^{h_{i}} \eta_{i}^{l}\left(\zeta_{i q}(t)\right)\left(C_{i}^{1} \bar{F}_{l}+C_{i}^{2} \bar{E}_{l}\right) \bar{x}_{i}(\phi) d \phi\right\} \\
\bar{f}_{i}(t)=\bar{x}_{i}(t)-\bar{x}_{i}(0), \bar{x}_{i}(t)=\left[x_{i}^{T}(t), u_{i}^{T}(t)\right], C_{i}^{1}=\left[I_{n}, 0_{n \times m}\right]^{T}, C_{i}{ }^{2}=\left[0_{m \times n}, I_{m}\right]^{T}, \bar{F}_{i}^{l}=\left[F_{i}^{l}, G_{2 i}{ }^{l}\right], \\
\bar{A}=\left[A_{i}^{x}, A_{i}^{u}\right], \bar{E}_{i}^{l}=\left[E_{i}^{l}, H_{i}^{l}\right]
\end{gathered}
$$

One can draw the following two closed loop control system equation:

$$
\begin{aligned}
& \dot{x}_{i}(t)=\hat{f}\left(x_{i}, u_{i}\right)=\sum_{l=1}^{r_{i}} \eta_{i}^{l}\left(\zeta_{i q}(t)\right)\left(F_{i}^{l} x_{i}(t)+G_{1 i}{ }^{l} w_{i}(t)+G_{2 i}{ }^{l} u_{i}(t)+\sum_{j=1 j \neq i}^{N} H_{j i} x_{j}(t)\right) \\
& \dot{\mathrm{u}}_{i}(\mathrm{t})=\hat{\mathrm{g}}\left(x_{i}, u_{i}\right)=\sum_{l=1}^{r_{i}} \eta_{i}^{l}\left(\zeta_{i q}(t)\right)\left(E_{i}^{l} x_{i}(t)+G_{1 i}{ }^{l} w_{i}(t)+H_{i}{ }^{l} u_{i}(t)+\sum_{j=1 j \neq i}^{N} H_{j i} x_{j}(t)\right)
\end{aligned}
$$

The above two equations are equivalent to:

$$
\dot{\bar{x}}_{i}(t)=\sum_{l=1}^{r_{i}} \eta_{i}{ }^{l}\left(\zeta_{i q}(t)\right)\left[\left(C_{i}^{1} \bar{F}_{i}^{l}+C_{i}^{2} \bar{E}_{i}^{l}\right) \bar{x}_{i}(t)+\sum_{j=1 j \neq i}^{N} H_{j i} x_{j}(t)+G_{1 i}{ }^{l} w_{i}(t)\right]
$$

If the design of closed-loop control equation (27) is reasonable, then the stability of sliding mode controller can be guaranteed

\subsection{The Analysis of Sliding Mode Stability}

It has been given the reliability proof of nonlinear system's (1) and equivalent system's (14) sliding mode surface. In order to let integral sliding mode run more stable, one shall meet the following requirements:

$$
\begin{aligned}
\dot{S}_{i}(t)= & \sum_{l=1}^{r_{i}} \eta_{i}{ }^{l}\left(\zeta_{i q}(t)\right) A_{i}{ }^{x}\left(\left(F_{i}^{l}+\Delta F_{i}^{l}\right) x_{i}(t)+G_{1 i}{ }^{l} w_{i}(t)+\left(G_{2 i}{ }^{l}+\Delta G_{2 i}{ }^{l}\right) u_{i}(t)+\sum_{j=1 j \neq i}^{N} H_{j i} x_{j}(t)\right)+A_{i}^{u} \dot{u}_{i}(t) \\
& -\sum_{l=1}^{r_{i}} \eta_{i}{ }^{l}\left(\zeta_{i q}(t)\right)\left[A_{i}{ }^{x}\left(F_{i}{ }^{l} x_{i}(t)+G_{2 i}{ }^{l} u_{i}(t)\right)+A_{i}{ }^{u}\left(E_{i}{ }^{l} x_{i}(t)+H_{i}{ }^{l} u_{i}(t)\right)\right]=0
\end{aligned}
$$


From (28) that the models of integral sliding control equation should be:

$$
\dot{u}_{i}(t)=\sum_{l=1}^{r_{i}} \eta_{i}^{l}\left(\zeta_{i q}(t)\right)\left[\left(E_{i}^{l} x_{i}(t)+H_{i}{ }^{l} u_{i}(t)\right)-\left(A_{i}^{u}\right)^{-1} A_{i}^{x}\left(\Delta F_{i}^{l} x_{i}(t)+\Delta G_{2 i}{ }^{l} u_{i}(t)+G_{1 i}{ }^{l} w_{i}(t)+\sum_{j=1 j \neq i}^{N} H_{j i} x_{j}(t)\right)\right]
$$

We can get the follow equation from (14), (29):

$$
\begin{aligned}
\dot{\bar{x}}_{i}(t) & =\sum_{l=1}^{r_{i}} \eta_{i}^{l}\left(\zeta_{i q}(t)\right)\left[\left(C_{i}^{1} \bar{F}_{i}^{l}+C_{i}^{2} \bar{E}_{i}^{l}+\left(C_{i}^{1}-C_{i}^{2}\left(A_{i}^{u}\right)^{-1} A_{i}^{x}\right) \Delta \bar{F}_{i}^{l}\right) \bar{x}_{i}(t)\right. \\
& \left.+\left(C_{i}^{1}-C_{i}{ }^{2}\left(A_{i}^{u}\right)^{-1} A_{i}^{x}\right)\left(\sum_{j=1 j \neq i}^{N} H_{j i} x_{j}(t)+G_{1 i}{ }^{l} w_{i}(t)\right)\right]
\end{aligned}
$$

Theorem 2: There exit a positive definite matrix $\mathrm{P}$ and a set of positive constant $\varepsilon_{l}$, if meeting the following LMI matrix, and one can make (30) in a compact region is semi-globally asymptotically stable.

$$
\left[\begin{array}{cccc}
\Omega_{l} & * & * & * \\
C_{i}^{1 T} P & \Theta_{l} & * & * \\
C_{i}^{2 T} P & 0 & -C_{i}^{2 T} P C_{i 2} & * \\
\varepsilon_{f} I_{(m+n)} & 0 & 0 & -\varepsilon_{l} I_{(m+n)}
\end{array}\right]<0
$$

$\Omega_{l}=P\left(C_{i}^{1} \bar{F}_{i}^{l}+C_{i}^{2} \bar{E}_{i}^{l}\right)+\left(C_{i}^{1} \bar{F}_{i}^{l}+C_{i}^{2} \bar{E}_{i}^{l}\right)^{T} P, \Theta_{l}=\left(C_{i}^{1}\right)^{T} P C_{i}^{1}-\varepsilon_{l} I_{n}$

The sliding mode surface matrix is given as follows: $\bar{S}_{i}=\left(C_{i}^{2}\right)^{T} P$

Proof: It's obtained that the nonlinear system (1) can be expressed by the uncertain T-S fuzzy model in (8) in a compact region. If the sliding motion of the closed-loop control system involving the uncertain T-S fuzzy model in (8), the sliding motion of the closed-loop control system involving the original nonlinear system[6] can be shown to be semi-globally asymptotically[7] stable.

Given the following Lyapunov candidate:

$$
V(\bar{x}(t))=\sum_{i=1}^{N} \bar{x}_{i}^{T}(t) P \bar{x}_{i}(t)
$$

Combining(31)and(32), one will have :

$$
\begin{aligned}
\dot{V}(\bar{x}(t)) & =\sum_{i=1}^{N} \sum_{l=1}^{r_{i}} \eta_{i}^{l}\left(\zeta_{i q}(t)\right) 2 \bar{x}_{i}^{T}(t) P\left[\left(C_{i}^{1} \bar{F}_{i}^{l}+C_{i}^{2} \bar{E}_{i}^{l}+\left(C_{i}^{1}-C_{i}^{2}\left(A_{i}^{u}\right)^{-1} A_{i}^{x}\right) \Delta \bar{F}_{i}^{l}\right) \bar{x}_{i}(t)\right. \\
& \left.+\left(C_{i}^{1}-C_{i}{ }^{2}\left(A_{i}^{u}\right)^{-1} A_{i}^{x}\right)\left(\sum_{j=1}^{N} H_{j \neq i} x_{j}(t)+G_{1 i}^{l} w_{i}(t)\right)\right]
\end{aligned}
$$

$\mathrm{P}$ is a positive definite matrix. Assuming that there is a matrix $M>0$, exist $P=M * M$ and $M=P^{\frac{1}{2}}$

$$
\begin{gathered}
-2 P C_{i}^{2}\left(A_{i}^{u}\right)^{-1} A_{i}{ }_{i}^{x} \Delta \bar{F}_{i}^{l}=-2 P C_{i}^{2}\left(\left(C_{i}^{2}\right)^{T} P C_{i}^{2}\right)^{-1}\left(C_{i}^{2}\right)^{T} P C_{i}^{1} \Delta \bar{F}_{i}^{l} \\
\leq P C_{i}^{2}\left(\left(C_{i}^{2}\right)^{T} P C_{i}^{2}\right)^{-1}\left(C_{i}^{2}\right)^{T} P C_{i}^{2}\left(\left(C_{i}^{2}\right)^{T} P C_{i}^{2}\right)^{-1}\left(C_{i}^{2}\right)^{T} P+\left(\Delta \bar{F}_{i}^{l}\right)^{T}\left(C_{i}^{1}\right)^{T} P C_{i}^{1} \Delta \bar{F}_{i}^{l} \\
\quad=P C_{i}^{2}\left(\left(C_{i}^{2}\right)^{T} P C_{i}^{2}\right)^{-1}\left(C_{i}^{2}\right)^{T} P+\left(\Delta \bar{F}_{i}^{l}\right)^{T}\left(C_{i}^{1}\right)^{T} P C_{i}^{1} \Delta \bar{F}_{i}^{l} \\
\dot{V}(\bar{x}(t)) \leq \sum_{i=1}^{N} \sum_{l=1}^{\eta_{i}} \eta_{i}^{l}\left(\zeta_{i q}(t)\right) \bar{x}_{i}^{T}(t)\left[\Omega_{l}+P C_{i}^{1} \Delta \bar{F}_{i}^{l}+\left(\Delta \bar{F}_{i}^{l}\right)^{T}\left(C_{i}^{1}\right)^{T} P+\left(\Delta \bar{F}_{i}^{l}\right)^{T}\left(C_{i}^{1}\right)^{T} P C_{i}^{1} \Delta \bar{F}_{i}^{l}\right. \\
\left.\left.+P C_{i}^{2}\left(\left(C_{i}^{2}\right)^{T} P C_{i}^{2}\right)^{-1}\left(C_{i}^{2}\right)^{T} P\right)+\left(C_{i}^{1}-C_{i}^{2}\left(A_{i}^{u}\right)^{-1} A_{i}^{x}\right)\left(\sum_{j=1 j \neq i}^{N} H_{j i} x_{j}(t)+G_{l i}{ }^{l} w_{i}(t)\right)\right] \bar{x}_{i}(t)
\end{gathered}
$$


In order to set up $\dot{V}(\bar{x}(t))<0$, we will establish the following formula (36)

$$
\begin{aligned}
& \Omega_{l}+P C_{i}^{1} \Delta \bar{F}_{i}^{l}+\left(\Delta \bar{F}_{i}^{l}\right)^{T}\left(C_{i}^{1}\right)^{T} P+\left(\Delta \bar{F}_{i}^{l}\right)^{T}\left(C_{i}^{1}\right)^{T} P C_{i}^{1} \Delta \bar{F}_{i}^{l}+P C_{i}^{2}\left(\left(C_{i}^{2}\right)^{T} P C_{i}^{2}\right)^{-1}\left(C_{i}^{2}\right)^{T} P \\
& +\left(C_{i}^{1}-C_{i}^{2}\left(A_{i}^{u}\right)^{-1} A_{i}^{x}\right)\left(\sum_{j=1 j \neq i}^{N} H_{j i} x_{j}(t)+G_{1 i}{ }^{l} w_{i}(t)\right)<0
\end{aligned}
$$

Because all child nodes' link and the disturbance are small comparing to the overall state and the control of the network, so here one can ignore the link and disturbance between child nodes. So formula(36)is equivalent to (37).

$$
\Omega_{l}+P C_{i}^{1} \Delta \bar{F}_{i}^{l}+\left(\Delta \bar{F}_{i}^{l}\right)^{T}\left(C_{i}^{1}\right)^{T} P+\left(\Delta \bar{F}_{i}^{l}\right)^{T}\left(C_{i}^{1}\right)^{T} P C_{i}^{1} \Delta \bar{F}_{i}^{l}+P C_{i}^{2}\left(\left(C_{i}^{2}\right)^{T} P C_{i}^{2}\right)^{-1}\left(C_{i}^{2}\right)^{T} P<0
$$

Formula (36) is equivalent to the following formula:

$$
\xi^{T}\left[\begin{array}{cc}
\Omega_{l}+P C_{i}^{2}\left(\left(C_{i}^{2}\right)^{T} P C_{i}^{2}\right)^{-1}\left(C_{i}^{2}\right)^{T} P & * \\
\left(C_{i}^{1}\right)^{T} P & \left(C_{i}^{1}\right)^{T} P C_{i}^{1}
\end{array}\right] \xi<0
$$

If considering the approximation error, the matrix can be redefined as follows:

$$
\xi^{T}\left[\begin{array}{cc}
-\varepsilon_{f}^{2} I_{(m+n)} & * \\
0 & I_{n}
\end{array}\right] \xi<0
$$

$T_{0} \cdots T_{p}$ are symmetric matrices. If there have $\tau_{1} \geq 0, \cdots \tau_{P} \geq 0$, then one has $T_{0}-\sum_{i=1}^{P} \tau_{i} T_{i}>0$ and $\eta^{T} T_{i} \eta \geq 0 \quad i=1, \cdots P$. A series of positive constant $\varepsilon_{l}$ have been taken into account:

$$
\left[\begin{array}{lc}
\Omega_{l}+P C_{i}^{2}\left(\left(C_{i}^{2}\right)^{T} P C_{i}^{2}\right)^{-1}\left(C_{i}^{2}\right)^{T} P+\frac{\varepsilon_{f}^{2}}{\varepsilon_{l}} I_{(m+n)} & * \\
\left(C_{i}^{1}\right)^{T} P & \left(C_{i}^{1}\right)^{T} P C_{i}^{1}-\varepsilon_{f} I_{n}
\end{array}\right]<0
$$

It follows that the formula (31) is equivalent to (39). The sliding motion of the integral sliding surface is semi-globally asymptotically stable if the LMIs in (31)are satisfied. And the proof is thus completed.

\section{Simulation Study}

One of the benchmark examples to demonstrate the performance of nonlinear control schemes is the control of an inverted pendulum. Consider the inverted pendulum system. $x_{1}(t)$ denotes the pendulum's angle and $x_{2}(t)$ denotes it's angular velocity. $g=9.8 \mathrm{~m} / \mathrm{sec} 2 \mathrm{is}$ the acceleration due to gravity, $\mathrm{M}$ is the mass of the cart, $\mathrm{m}$ is the mass of the pole, 21 is the pole's length, and $u$ is the control force. The system's dynamical equations are:

$$
\left\{\begin{array}{l}
\dot{x}_{1}=x_{2} \\
\dot{x}_{2}=F\left(x_{1}, x_{2}\right)+G\left(x_{1}, x_{2}\right) u
\end{array}\right.
$$




$$
\left\{\begin{array}{l}
F\left(x_{1}, x_{2}\right)=\frac{g \sin x_{1}-\frac{m l x_{2}^{2} \cos x_{1} \sin x_{1}}{m+M}}{l\left(\frac{4}{3}-\frac{m \cos ^{2} x_{1}}{m+M}\right)} \\
G\left(x_{1}, x_{2}\right)=\frac{\frac{\cos x_{1}}{m+M}}{l\left(\frac{4}{3}-\frac{m \cos ^{2} x_{1}}{m+M}\right)}
\end{array}\right.
$$

The membership functions are shown as follow:

$$
\begin{gathered}
A_{1}=\left[\begin{array}{cc}
0 & 1 \\
16.976 & 0
\end{array}\right] A_{2}=\left[\begin{array}{cc}
0 & 1 \\
5.678 & 0
\end{array}\right] A_{3}=\left[\begin{array}{cc}
0 & 1 \\
0.415 & 0
\end{array}\right] \\
B_{1}=\left[\begin{array}{c}
0 \\
-0.1693
\end{array}\right] \quad B_{2}=\left[\begin{array}{c}
0 \\
-0.0803
\end{array}\right] \quad B_{3}=\left[\begin{array}{c}
0 \\
-0.0049
\end{array}\right]
\end{gathered}
$$

By giving formula (31), (37) with $\varepsilon_{f}$ being chosen as 0.03 , the corresponding siding surface matrix and the controller gains can be given as follows:

$$
\begin{aligned}
& \bar{K}_{1}=[24976.3276,23748.9374,129.8720], \bar{K}_{2}=[12996.3129,12399.4569,-70.1476] \\
& \bar{K}_{3}=[820.3965,790.1849,-4.4192], \bar{S}=[-0.5136,-0.5375,0.0031]
\end{aligned}
$$

A number of simulations of the closed-loop control system consisting of the original nonlinear system and the fuzzy dynamic integral sliding-mode controller have carried out and it's found that the pendulum can be balanced from $x_{1} \in\left[-85.6^{\circ}, 85.6^{\circ}\right]$.The closed-loop control system's state trajectories simulations and control input simulations are given as follows:

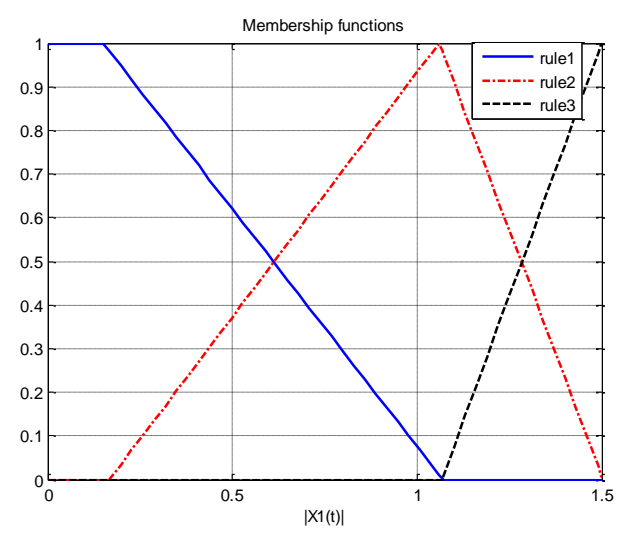

Figure 1. Membership Function 


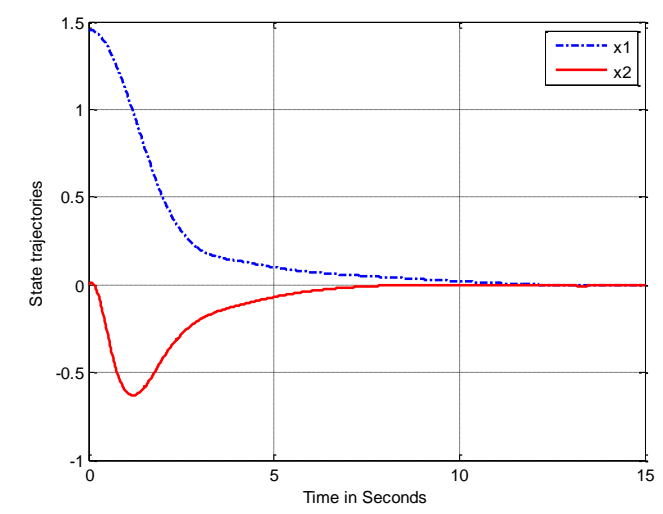

Figure 2. State Trajectories

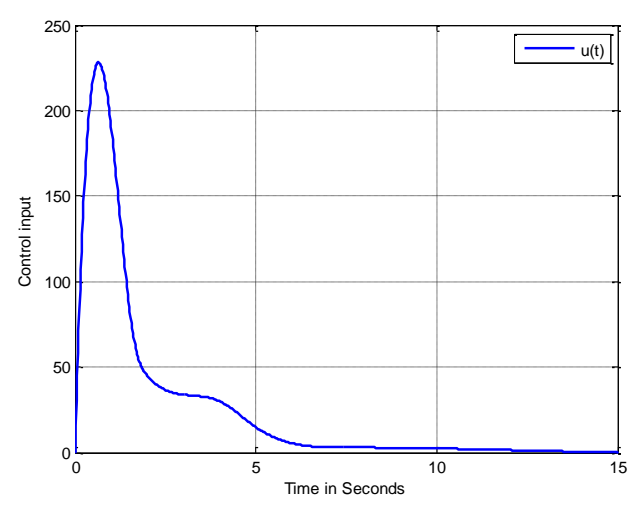

Figure 3. Control Input

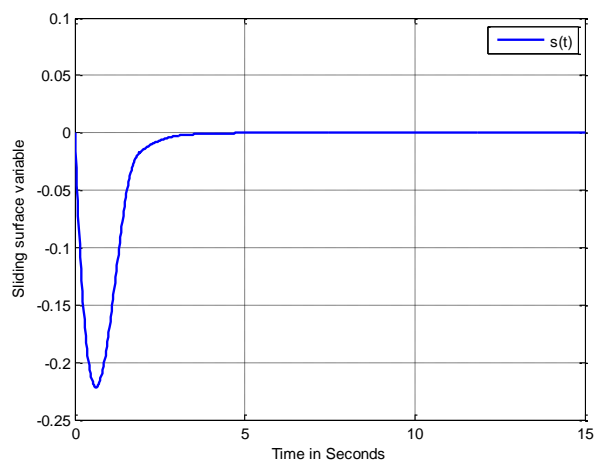

Figure 4. The Value of $\mathrm{S}(\mathrm{T})$

\section{Conclusion}

A novel dynamic integral sliding mode control scheme has been developed for complex networks systems based on T-S fuzzy models. It has been shown that the sliding mode can be achieved and maintained almost surely since the initial time with the integral sliding surface and a novel dynamic sliding-mode controller. And sufficient conditions to guarantee the stability of the sliding motion are given in terms of LMIs. Simulation results are provided advantages of the approaches proposed in this paper. 


\section{References}

[1] D. D. Siljac, "Decentralized Control of Complex Systems [M]", Boston: Academic Press, (1991).

[2] D. W. C. Ho and Y. Niu, "Robust Fuzzy Design for Nonlinear Uncertain Stochastic Systems via Sliding-mode Control", IEEE Transactions on Fuzzy Systems, 15, 350 (2007).

[3] V. I. Utkin, "Variable Structure Systems with Sliding Modes [J]", IEEE Transactions on Automatic Control, 22, 212 (1977).

[4] V. I. Utkin, J. Guldner and J. Shi, "Sliding Mode Control in Electromechanically Systems [M]", London: Taylor \& Francis, (1999).

[5] Y. Q. Xia, J. Chen and G. P. Liu, "Robust Adaptive Sliding Mode Control for Uncertain Time-delay Systems [J]", International Journal of Adaptive Control and Signal Processing, 23, 863 (2009).

[6] G. Qing, L. Lu and F. Gang, "Universal Fuzzy Integral Sliding-Mode Controllers Based on T-S Fuzzy Models [J]", IEEE Transitions on fuzzy systems, (2013).

[7] X. R. Liu, "Research on the H1 Control of Nonlinear Interconnected Large-Scale Systems based on Fuzzy Models [D]", Northeastern University, (2006).
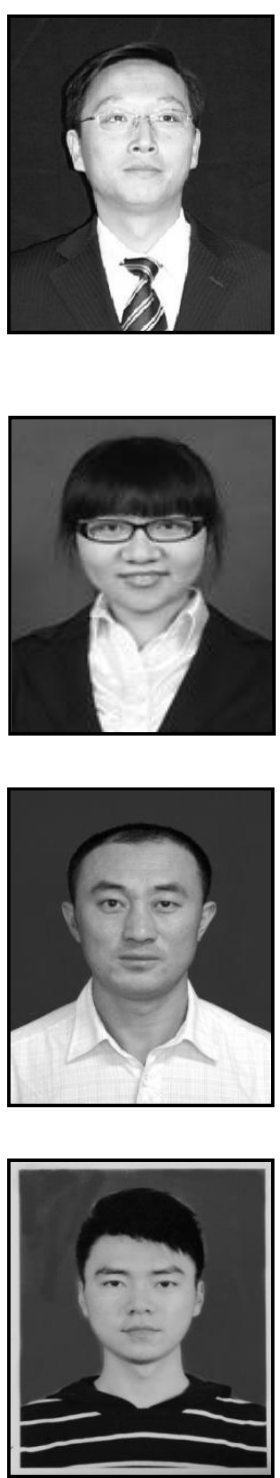

\section{Authors}

Dongsheng Yang, Liaoning Province, China, in1977. He received the B.S. degree, M.S. degree, and Ph. D. degree from Northeastern University, Shenyang, China, in 1999, 2004, and2007, respectively. Since 2004, he has been with the Northeastern University. His research interests include fuzzy control and fuzzy systems, chaotic control, and complex systems.

Bingqing Li, She received the B.E. degree in Electrical engineering and automation from Northeastern University, Liaoning, China, in 2013, where she is currently working towards the M.S. degree in electrical engineering. Her research interests include fuzzy control, scheduling of electrical power, and mart power grid.

\section{He Jiang}

He received the B.E. degree in automation from Northeastern University, Liaoning, China, in 2014, where he is currently working towards the Ph.D. degree in control theory and control engineering. His research interests include fuzzy control, robust control, and complex dynamical network control.

Xingyu Liu, He is the director of sales department's marketing department at state grid Liaoning power company, Shenyang, China. His research interests include scheduling of electrical power, smart power grid, planning, energy management and grey theory. 


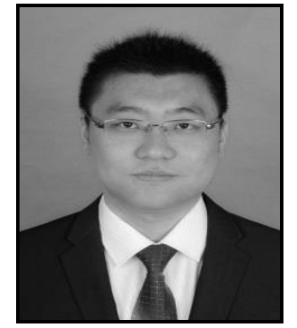

Yihe Wang, He comes from state grid Shenyang electric power supply company, Shenyang, China. His research interests include complex dynamical network control, fuzzy control, and power Planning. 